\title{
A new probe of black holes and their environment: Luminous flares from tidally disrupted stars
}

\author{
S. Komossa \\ Max-Planck-Institut für Radioastronomie, Auf dem Hügel 69, 53121 Bonn, Germany \\ skomossa@mpifr-bonn.mpg.de
}

\begin{abstract}
The luminous accretion flares from tidally disrupted stars represent a powerful probe of the presence of supermassive black holes (SMBHs) in otherwise non-active galaxies, of accretion physics and BH spin, of jet formation, and relativistic effects. Further, the reprocessing of the continuum radiation of the flare into IR, optical and UV emission lines provides us with multiple new diagnostics of the properties of any gaseous material in the vicinity of the SMBH and in the host galaxy itself. While first events were discovered in the course of the ROSAT allsky survey in X-rays, the last few years have seen the detection of several more flares, including in the UV, optical and radio band and via their emission-line "echoes". A wealth of applications will become feasible in upcoming years, once flares are detected in large numbers in current and future sky surveys.
\end{abstract}

Keywords. Galaxies: nuclei - galaxies: active - galaxies: emission-lines - black holes

\section{Introduction}

The tidal disruption, and subsequent accretion, of a star by a supermassive black hole produces a luminous flare of electromagnetic radiation, detectable out to large cosmic distances. About 25 events have been seen so far; initially found in the form of luminous X-ray flares from otherwise non-active galaxies during the ROSAT all-sky survey (RASS), and more recently also in the UV, optical, hard X-ray and radio band. Upcoming transient surveys may detect these events by the 1000s, opening up a new window to study accretion processes and the immediate environment of supermassive black holes.

A star is disrupted once the tidal forces exerted by the supermassive black hole exceed the self-gravity of the star. In the course of the whole process, i.e., the disruption, ejection and accretion of the stellar material, there are several sources of radiation (Fig. 1):

- After disruption, a fraction of the stellar material is accreted onto the SMBH, producing an accretion flare which can be as bright as the Eddington luminosity. It will last for weeks, then decline on the timescale of months to years, with a decline law scaling as $L \propto t^{-5 / 3}$ in X-rays. In most cases, this accretion phase is the brightest phase of the tidal disruption event (TDE).

- During the accretion phase, a (radio) jet may be launched.

- In case of a deeply plunging stellar orbit, squeezing of the star can be extreme. If nuclear burning is ignited, this would result in a GRB-like explosion.

- Part of the stellar material will escape into unbound orbits, and collision with surrounding interstellar matter could cause an event which shares similarity with a supernova.

- Stream-stream collisions of the returning stellar debris, which was spread across a range of bound orbits, will cause shocks. 
- A partially disrupted compact object, like a neutron star or white dwarf, is a strong source of gravitational waves $(\mathrm{GW})$ as they will be detectable with future space-based GW observatories.

- The luminous continuum emission produced during the accretion phase photoionizes ambient gaseous material and will be reprocessed into IR-optical-UV emission lines, causing an emission-line "light-echo" with a time delay which reflects the light-travel time, and the density and properties of the surrounding medium.

\section{tidal disruption of stars - sources of radiation}

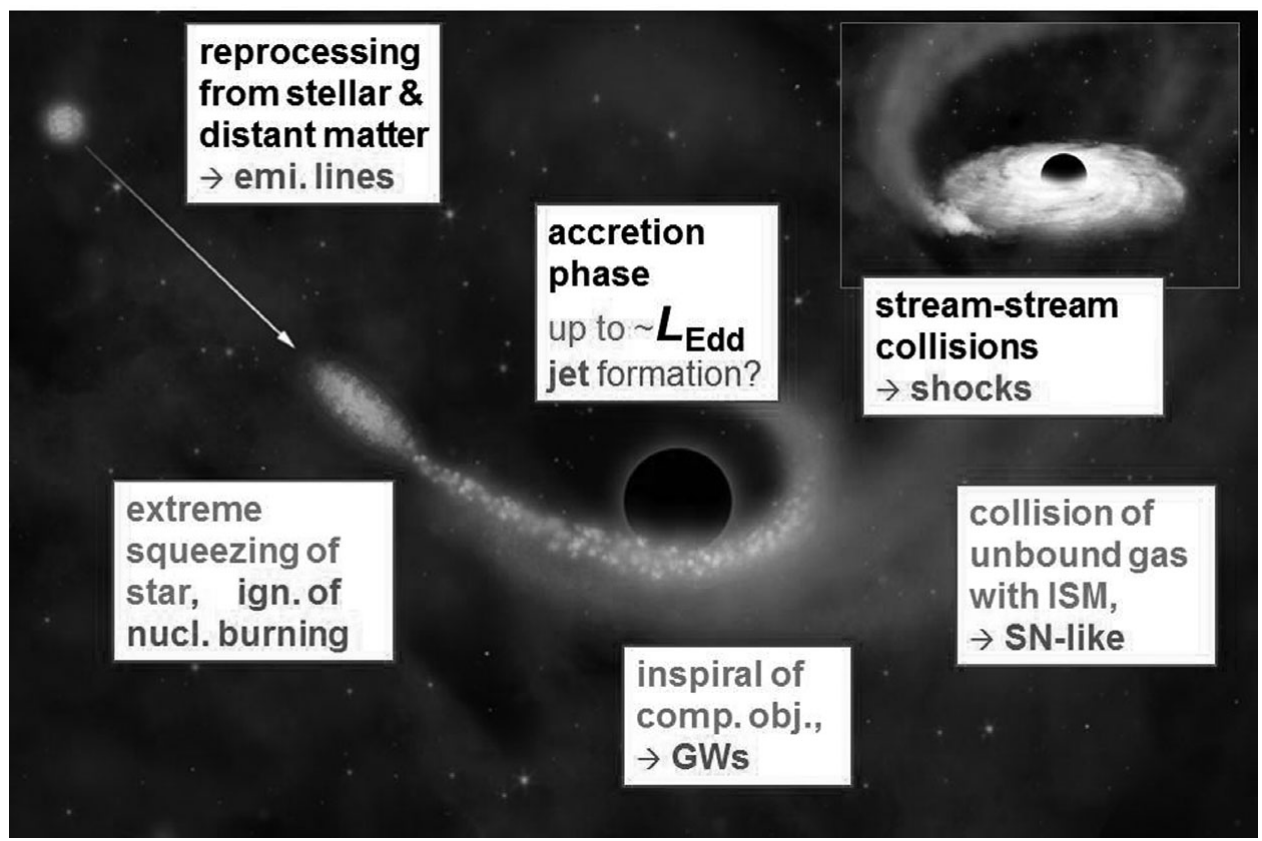

Figure 1. Sources of radiation during the tidal disruption of a star by a supermassive black hole. The brightest phase is the accretion phase.

\section{Tidal disruption events in X-rays}

The predicted rates of TDEs are low, on the order of $10^{-4 \ldots-5} / \mathrm{yr} /$ galaxy. Therefore, large- or all-sky survey type experiments are best suited to detect these events. Since the accretion phase produces a luminous flare, with peak in the EUV or soft X-rays, TDEs are best detected in X-rays. Further, in X-rays the contrast with respect to the (faint) permanent emission of the host is highest, and X-rays trace the immediate vicinity of the $\mathrm{BH}$, opening up new possibilities of probing accretion near the event horizon and relativistic effects (Sect. 3).

The ROSAT all-sky survey was an ideal experiment to detect these events. Indeed, TDEs were first seen with ROSAT, and more recently similar X-ray events have also been found with Chandra, XMM-Newton and Swift. Essentially all of them share similar properties, and in particular:

- Huge (soft) X-ray peak luminosities, reaching almost up to $10^{45} \mathrm{erg} / \mathrm{s}$.

- Amplitudes of decline up to factors 1000-6000, more than a decade after their observed high-states. 
- Very soft X-ray spectra during the observed high-states $\left(k T_{\mathrm{BB}} \sim 0.04-0.1 \mathrm{keV}\right)$, followed by a spectral hardening on the time scale of years.

- Their host galaxies show essentially no evidence for permanent activity as seen in active galactic nuclei (AGN). Years after the flare, the galaxies are optically inactive, radio inactive, and $\mathrm{X}$-ray inactive.

- X-ray lightcurves show a rapid rise (in the few cases, where pre-flare observations exist) $\dagger$, then a decline on the timescale of months-years, consistent with the law $L \propto t^{-5 / 3}$ predicted by tidal disruption theory.

All these properties agree remarkably well with theoretical predictions (e.g., Rees 1988).

\section{Tidal disruption events as probes of strong gravity}

Upcoming X-ray surveys will detect more than 100s or 1000s of TDEs. Once followed up rapidly right after discovery, by deep pointed X-ray and multi-wavelength observations, TDEs will provide us with new probes of strong gravity, accretion physics and jet formation:

- Relativistic iron lines forming and evolving in the temporary accretion disk will probe GR.

- Precession effects map the Kerr metric.

- Black hole spin affects the iron line profile, and determines the maximum black hole mass for which any star can be disrupted, and therefore also disruption rates depend on spin. Once these quantities are measured well, they allow to determine or constrain the spin of the SMBH.

- Accretion-disk coronae: while flares are initially very X-ray soft, a hard spectral component develops with time, first seen in ROSAT spectra of NGC 5905. This hard component may indicate the development of a disk corona, and its formation can thus be studied with TDEs.

\section{Emission-line reprocessing: imagine we could map a whole galaxy core, following one giant flare}

Tidal flares which occur in gas-rich galaxies will provide us with a powerful new tool of performing reverberation mapping of the cores of these galaxies. When the luminous continuum radiation of the flare travels across the galaxy core, it will photoionize any circumnuclear material and is reprocessed into line radiation. This emission-line "light echo" enables us to perform "reverberation mapping" of any gaseous material that is present in the galaxy core. This will, in principle, include the broad-line region (if present), the molecular torus, and the narrow-line region, and will also allow us to determine, which of these regions permanently exist in non-active galaxies. The emission-line fluxes, line widths, line shifts, and their evolution with time, tightly constrain the amount, density, composition, dynamics and geometry of the material.

Thanks to the Sloan Digital Sky Survey (SDSS; York et al. 2000) archive, and based on a systematic search, several candidate TDE emission-line echoes have been identified in the last few years (Komossa et al. 2008, 2009, Wang et al. 2011, 2012). They are characterized by luminous, transient high-ionization emission lines including transitions from [FeVII]-[FeXIV], HeII, and Balmer lines of very unusual and variable profile (Fig. 2).

$\dagger$ NGC 5905 shows a rise to its observed maximum over a period of 5 days corresponding to the time interval when it was covered by the RASS (Komossa \& Bade 1999). 


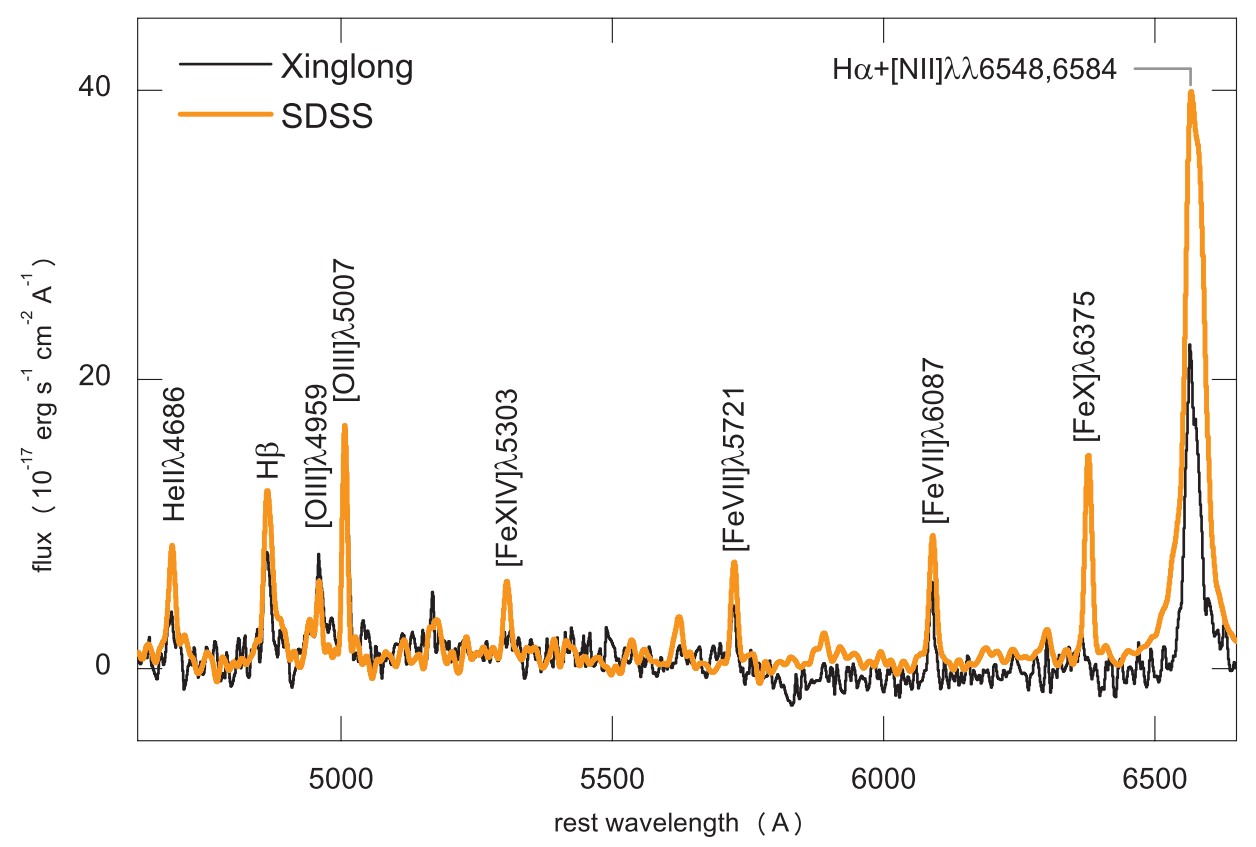

Figure 2. Light echo of a huge flare: Transient iron emission lines in the optical spectrum of SDSSJ 095209.56+214313.3. While the highest ionization iron lines were very bright in the initial SDSS spectrum (orange), they were no longer detected in a Xinglong spectrum (black) taken several years later (see Komossa et al. 2008 for details).

Intrinsically luminous, the high-ionization lines fade dramatically on the timescale of years. These lines have been excited by a luminous flares; yet the host galaxies of most of these events show little, if any, evidence for ongoing classical AGN activity. TDEs therefore provide a good explanation of the observed spectral features. Future detection of similar events, combined with dense spectroscopic sampling of the emission-line evolution, will provide us with a new means of reverberation-mapping the cores of (otherwise nonactive) galaxies, following one giant (tidal disruption) flare.

Upcoming spectroscopic surveys like the one carried out with LAMOST will find many more emission-line signatures of TDEs.

\section{References}

Komossa, S. \& Bade, N. 1999, Astron. Astrophy., 343, 775

Komossa, S., et al. 2008, ApJ, 678, L13

Komossa, S., et al. 2009, ApJ, 701, 105

Rees, M. J. 1988, Nature, 33, 523

Wang, T., et al. 2011, ApJ, 740, 85

Wang, T., et al. 2012, ApJ, 749, 115

York, D. G., et al. 2000, AJ, 120, 1579 